\title{
ESTIMATION OF SPICULE MAGNETIC FIELD USING OBSERVED MHD WAVES BY THE HINODE SOT
}

\author{
Yeon-Han Kim ${ }^{1}$, Su-Chan Bong ${ }^{1}$, Young-Deuk Park ${ }^{1}, \mathrm{Kyung}$-Suk Cho ${ }^{1}$, \\ YONG-JAE MOON ${ }^{2}$, AND YoshinORI SuEMATSU ${ }^{3}$ \\ ${ }^{1}$ Korea Astronomy and Space Science Institute, 36-1 Hwaam, Yusong, Daejeon 305-348, Korea \\ E-mail: yhkim@kasi.re.kr \\ 2 Department of Astronomy and Space Science, Kyunghee University, Yongin 446-701, Korea \\ 3 National Astronomical Observatory of Japan, 2-21-1 Osawa, Mitaka, Tokyo 181-8588, Japan \\ (Received October 31, 2008; Accepted December 8, 2008)
}

\begin{abstract}
Using the MHD coronal seismology technique, we estimated the magnetic field for three spicules observed in 2008 June. For this study, we used the high resolution Ca II H line (3968.5 $\AA)$ images observed by the Hinode SOT and considered a vertical thin flux tube as a spicule model. To our knowledge, this is the first attempt to estimate the spicule magnetic field using the Hinode observation. From the observed oscillation properties, we determined the periods, amplitudes, minimum wavelengths, and wave speeds. We interpreted the observed oscillations as MHD kink waves propagating through a vertical thin flux tube embedded in a uniform field environment. Then we estimated spicule magnetic field assuming spicule densities. Major results from this study are as follows : (1) we observed three oscillating spicules having durations of 5-7 minutes, oscillating periods of 2-3 minutes, and transverse displacements of $700-1000 \mathrm{~km}$. (2) The estimated magnetic field in spicules is about 10-18 G for lower density limit and about 43-76 G for upper density limit. (3) In this analysis, we can estimate the minimum wavelength of the oscillations, such as $60000 \mathrm{~km}, 56000 \mathrm{~km}$, and $45000 \mathrm{~km}$. This may be due to the much longer wavelength comparing with the height of spicules. (4) In the first event occurred on 2008 June 03, the oscillation existed during limited time (about $250 \mathrm{~s}$ ). This means that the oscillation may be triggered by an impulsive mechanism (like low atmospheric reconnection), not continuous. Being compared with the ground-based observations of spicule oscillations, our observation indicates quite different one, i.e., more than one order longer in wavelength, a factor of 3-4 larger in wave speed, and 2-3 times longer in period.
\end{abstract}

Key words : Sun: activity — Sun: chromosphere — Sun: magnetic field — Sun: oscillations

\section{INTRODUCTION}

Measurement of coronal magnetic field has been a challenge for solar physicists for several decades. There has been several efforts to measure the coronal magnetic filed (Nakariakov \& Ofman 2001 and references therein), e.g., radio methods based on gyroresonance emission, optical and EUV methods analyzing coronal emission spectrum modification due to the Zeeman and Hanle effects, Faraday rotation observations from radio sources which is proper for the upper part of corona, and indirect methods extrapolating the measured photospheric magnetic field. However, still now, there have been only limited cases to measure the coronal magnetic field with considerable accuracy.

The MHD coronal seismology is a newly developed method for the measurement of the coronal magnetic field from observed wave signatures in the corona (Nakariakov \& Ofman 2001). The idea of the method is similar to the helioseismology. We can observe wave

Corresponding Author: Y.-H. Kim properties and physical parameters of the medium, such as amplitudes, temporal and spatial spectra, and temperature and density structures. Then, assuming appropriate model for coronal structure, we can connect the observed properties with the unknown physical parameters such as magnetic field and transport coefficients based on the MHD wave theory. During SOHO and TRACE era, high-resolution observations of MHD waves in coronal loops (Aschwanden et al. 1999; Nakariakov et al. 1999; De Moortel et al. 2000; Robbrecht et al. 2001) and polar plumes (DeForest \& Gurman 1998; Ofman et al. 1999) provide unique opportunity for MHD coronal seismology. However, the source of these waves is uncertain up to date, although global p-modes in the photosphere are an obvious candidate.

Regarding the existence of MHD waves in the corona, the coronal MHD waves can be generated by near-by solar flares, magnetic reconnections, or photospheric disturbances which can reach up to the corona via magnetic field anchored in the photosphere. Spicules, jetlike chromospheric structures remarkably seen in $\mathrm{H} \alpha$ 
at the solar limb, probably are concentrated at the network boundaries which are regions between supergranule cells and having relatively enhanced magnetic fields (Suematsu 1998; Sterling 2000). Thus it is natural that the MHD wave propagation in the solar atmosphere may be traced through the oscillatory dynamics of spicules. Oscillations in spicules with $\sim 5$ minutes period have been detected by ground based and space observations (Kulidzanishvili \& Zhugzhda 1983; De Pontieu et al. 2003; Xia et al. 2005) and are probably connected with global p-modes. On the other hand, the shorter-period oscillations ( $\sim 1$ minute) have been reported by Nikolsky and Platova (1971) as oscillatory displacements of spicule axes at one particular height. If we consider a thin magnetic flux tube anchored in the photosphere as a spicule model, then the oscillatory motion of spicules can be interpreted by the propagation of kink waves. Moreover, it is well known that transverse kink waves can be generated in photospheric magnetic flux tube by buffeting of granular motions (Roberts 1979; Hollweg 1981; Spruit 1981; Hasan \& Kalkofen 1999).

Nakariakov \& Ofman (2001) developed a new method (MHD coronal seismology) for the determination of absolute magnetic field strength in coronal closed magnetic structures, based on the analysis of flare-generated oscillations of coronal loops observed by the TRACE. They estimated the magnetic field strength of coronal loops as 4-30 G. Recently, the observational signature of propagating kink waves in spicules has been reported by Kukhianidze et al. (2006). They reported periodic spatial distributions of Doppler velocities with height through spectroscopic analysis of $\mathrm{H} \alpha$ height series in solar limb spicules. Zaqarashvili et al. (2007) and Singh \& Dwivedi (2007) estimated the magnetic field strength in spicules using this observed kink wave as 8-16 G. Nakariakov \& Ofman (2001) also suggested that improved diagnostic of observational properties (e.g., the loop length, the oscillation period, and the plasma density in the loop) will significantly improve the precision of the MHD coronal seismology technique.

Recently, the Hinode (formerly called Solar-B; Kosugi et al. 2007), which is a Japanese solar mission for high-resolution observation from the photosphere to the corona, was launched on 2006 September 22 . The main purpose of Hinode is to address key questions in solar physics such as coronal heating, driving mechanism of explosive events, and source of the solar magnetic fields. Due to unprecedented high-resolution observations by the Hinode, many wave phenomena were observed in the solar atmosphere such as prominences (Okamoto et al. 2007), spicules (De Pontieu et al. 2007), and X-ray jets (Cirtain et al. 2007). In this work, we apply the MHD coronal seismology technique to three spicules in order to estimate their magnetic field strengths using high-resolution Hinode SOT data.

The paper is organized as follows. In Section II, we describe the method of MHD coronal seismology to estimate the magnetic field from the observed oscillation signatures. The observation and data analysis are explained in Section III. In Section IV, we present our results and finally summary and discussion are delivered in Section V.

\section{METHOD}

When we consider a vertical thin magnetic flux tube anchored in the photosphere, which is assumed to be embedded in a field-free environment. According to Roberts (2004), the propagation of kink waves in a vertical thin flux tube is governed by

$$
\frac{\partial^{2} u}{\partial t^{2}}=\frac{B_{0}^{2}}{\mu_{0}\left(\rho_{0}+\rho_{e}\right)} \frac{\partial^{2} u}{\partial z^{2}}+\frac{\left(\rho_{0}-\rho_{e}\right)}{\left(\rho_{0}+\rho_{e}\right)} g \frac{\partial u}{\partial z},
$$

where $u(z, t)$ is the transverse displacement of a tube, $\rho_{0}$ and $\rho_{e}$ are plasma densities inside and outside respectively, and $g$ is the acceleration due to gravity. The first term on the right-hand side of Eq. (1) is due to the magnetic tension force in the bent tube and the second term is due to the buoyancy force on the tube. Eq. (1) becomes

$$
\frac{\partial^{2} u}{\partial t^{2}}=C_{k}^{2} \frac{\partial^{2} u}{\partial z^{2}}+\frac{\left(\rho_{0}-\rho_{e}\right)}{\left(\rho_{0}+\rho_{e}\right)} g \frac{\partial u}{\partial z}
$$

where $C_{k}=\left(\frac{\rho_{0}}{\rho_{0}+\rho_{e}}\right)^{\frac{1}{2}} C_{A 0}$ is the kink speed in the tube and $C_{A 0}$ is the Alfvén wave speed. Neglecting the gravitational stratification, we get a simple dispersion relation as

$$
\omega^{2}=C_{k}^{2} k_{z}^{2}
$$

where $k_{z}$ and $\omega$ are the vertical wave number and the frequency of kink waves, respectively. Both are observable quantities.

By the way, if we allow an external magnetic field $B_{e}$, not field-free, the pressure balance between inside and outside the tube is

$$
p_{0}+\frac{B_{0}^{2}}{2 \mu_{0}}=p_{e}+\frac{B_{e}^{2}}{2 \mu_{0}},
$$

where $\mu_{0}$ is magnetic permeability. In this case, the kink speed $C_{k}$ can be rewritten as, which is given by Edwin \& Roberts (1983),

$$
C_{k}=\left(\frac{\rho_{0} C_{A 0}^{2}+\rho_{e} C_{A e}^{2}}{\rho_{0}+\rho_{e}}\right)^{\frac{1}{2}},
$$

where $C_{A e}$ is the Alfvén speed outside tube. In the case of low- $\beta$ plasma, Eq. (4) implies $\rho_{0} C_{A 0}^{2} \approx \rho_{e} C_{A e}^{2}$. Thus, Eq. (5) becomes

$$
C_{k}=\sqrt{2}\left(\frac{1}{1+\frac{\rho_{e}}{\rho_{0}}}\right)^{\frac{1}{2}} C_{A 0} .
$$




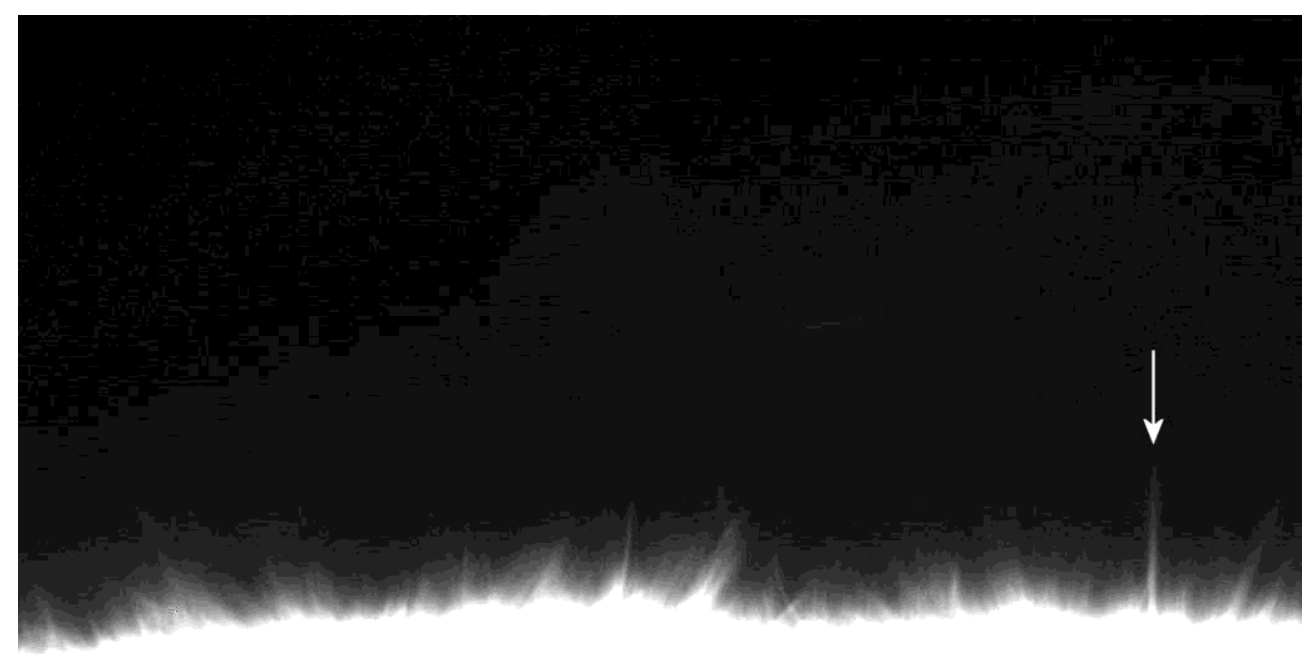

Fig. 1. - North polar limb of the Sun observed by the Hinode SOT. This is a Ca II H line image at 00:00:00 UT on 2008 June 03. The field of view of the image is $110 \times 55 \operatorname{arcsec}^{2}$. The white arrow indicates the first event of this study.

Then the magnetic field comes straight from Eq. (3) which is given by

$$
B_{0}=\sqrt{\frac{\mu_{0}}{2}} \frac{L}{P} \sqrt{\rho_{0}\left(1+\frac{\rho_{e}}{\rho_{0}}\right)},
$$

where $B_{0}$ is the magnetic field, $L$ is the wavelength, and $P$ is the period of the kink wave. In this study, we set $\frac{\rho_{e}}{\rho_{0}}=0.1$. Since the realistic density ratio $\frac{\rho_{e}}{\rho_{0}}$ is usually much smaller, it does not affect kink speed. Finally, if we know the density of a spicule, we can estimate the magnetic field strength from Eq. (7).

\section{OBSERVATION AND DATA ANALYSIS}

The Solar Optical Telescope (SOT; Tsuneta et al. 2008) aboard the Hinode satellite is composed of two parts: the Optical Telescope Assembly (OTA; Suematsu et al. 2008) and the Focal Plane Package (FPP). The OTA is a diffraction-limited Gregorian telescope with a diameter of $50 \mathrm{~cm}$ which is the largest solar optical telescope in space, and the FPP includes the Narrowband Filter Imager (NFI), the Broadband Filter Imager (BFI), and the Spectro-Polarimeter (SP). The SOT provides unprecedented high-resolution photometric and vector magnetic images of the photosphere and chromosphere with a very stable point spread function and is equipped with an image stabilization system (Shimizu et al. 2008) with the performance better than 0.01 arcsec rms. The BFI produces photometric images with broad spectral resolution in six bands (CN band, Ca II H line, G band, and three continuum bands) at the highest spatial resolution available from the SOT (0.0541 arcsec pixel ${ }^{-1}$ sampling) and at a rapid cadence $(<10$ s) over a $218 \times 109$ arcsec FOV. For this study, we used Ca II H line data of the BFI observed in 2008
June. We identified three spicules in the north polar region of the Sun. The images have a dimension of 1024 $\times 512$ pixels with a pixel size of 0.109 arcsec. The time cadence of data is 16 seconds.

Our data analysis procedures are as follows. (1) Using a standard calibration routine for SOT filtergrams in Solarsoft package ("fg_prep.pro"), we corrected the camera readout defects, the dark current, and flat fielding, and remove radiation belt/cosmic ray spikes and streaks. (2) We look for spicules having a quite long lifetime, a relatively higher structure, and an oscillatory behavior. (3) We make a time-slice image at a fixed position of the spicule axis. This time-slice image is basically the same as so-called "space-time plot" or "time-distance plot" already presented by many authors (e.g., De Pontieu et al. 2007; Okamoto et al. 2007; Cirtain et al. 2007). (5) We determine the parameters of spicule oscillations such as periods, amplitudes, transverse velocities, wavelengths, and wave speeds. Regarding the wavelength of spicule oscillations, we cannot determine the exact wavelength from the time-slice data of spicules. Thus we examined several time-slices obtained at different positions along the spicule axis. However, we cannot find out any phase differences among them. It may be due to the fact that the spicule lengths are too short to give a definite wavelength. In other words, there is a possibility that the wave associated with spicule oscillations has a long wavelength. In this case, we can only estimate the minimum wavelength of spicule oscillations and it depends on the time cadence of the data, the oscillation period, and the length of spicule. We will describe this problem more in the next section. (6) We estimate the wave speed from the estimated wavelength and the period. 
TABLE 1.

EVENT SUMMARY FOR THREE OSCILLATING SPICULES OBSERVED IN 2008 JUNE

\begin{tabular}{|c|c|c|c|c|c|c|c|}
\hline$\overline{\overline{\text { DATE }}}$ & $\begin{array}{c}\text { OBSERVATION } \\
\text { TIME } \\
\text { (UT) }\end{array}$ & $\begin{array}{c}\text { DURATION } \\
\text { (SEC) }\end{array}$ & $\begin{array}{c}\overline{P E R I O D} \\
(\mathrm{SEC})\end{array}$ & $\begin{array}{c}\text { AMPLITUDE } \\
(\mathrm{KM})\end{array}$ & $\begin{array}{l}\text { TRANSVERSE } \\
\text { VELOCITY } \\
\left(\mathrm{KM} \mathrm{S}^{-1}\right)\end{array}$ & $\begin{array}{c}\text { MINIMUM } \\
\text { WAVELENGTH } \\
(\mathrm{KM})\end{array}$ & $\begin{array}{c}\text { WAVE SPEED } \\
\left(\mathrm{KM} \mathrm{S}^{-1}\right)\end{array}$ \\
\hline JUNE 03 & $\begin{array}{c}00: 00: 03 \\
-00: 07: 14\end{array}$ & 431 & 130 & 1000 & 15 & 60000 & 460 \\
\hline JunE 04 & $\begin{array}{c}23: 33: 33 \\
-23: 38: 52\end{array}$ & 320 & 180 & 700 & 8 & 56000 & 310 \\
\hline JuNE 04 & $\begin{array}{c}23: 42: 04 \\
-23: 47: 09\end{array}$ & 305 & 170 & 800 & 9 & 45000 & 260 \\
\hline
\end{tabular}

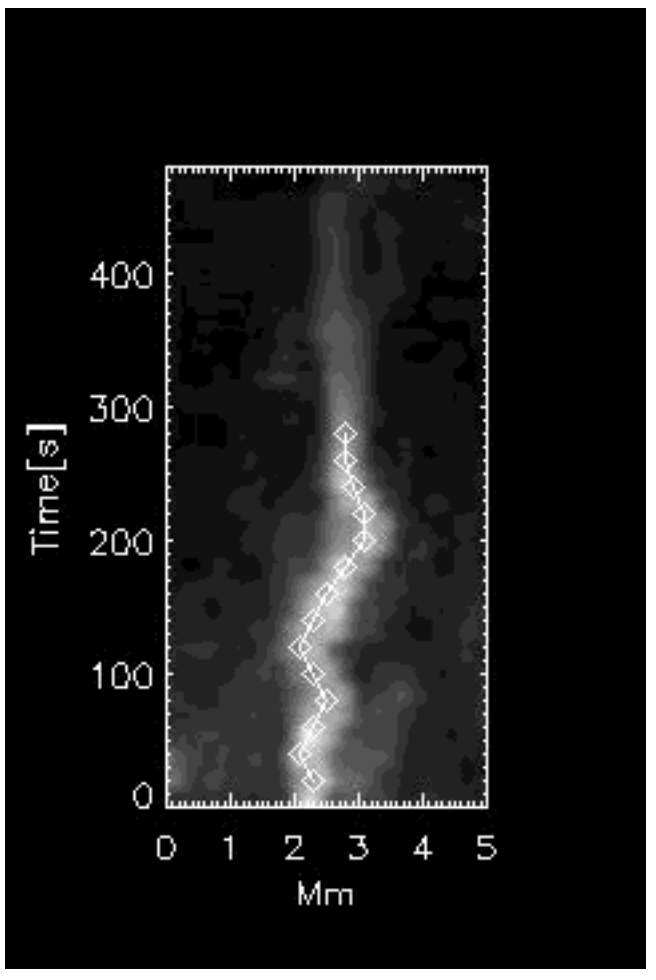

Fig. 2.- Time-slice image at the fixed position $(6000 \mathrm{~km}$ height) on the spicule axis. The spicule occurred at 00:00 UT on 2008 June 03.

\section{RESULTS}

Finally, we identified three oscillating spicules. Figure 1 shows the north polar limb of the Sun at 00:00 UT on 2008 June 03 and we can identify several spicules easily. Using a movie file, we can easily see ubiquitous horizontal oscillation signatures in the north polar limb of the Sun. In the rightmost side of the figure, there is a relatively tall spicule which is the first event of our study. In addition, we identified two more spicules on 2008 June 04 . The events are summarized in Table 1 which contains date, observing time, duration, oscillation period, minimum wavelength, and wave speed.

\section{(a) Spicule 1 (2008 June 03 00:00 UT)}

This spicule occurred during from 00:00:03 UT to 00:07:14 UT on 2008 June 03 in the north polar limb of the Sun. Figure 2 shows the time-slice image at the fixed position (6000 km height) of the spicule. Total duration is about $430 \mathrm{~s}$ and the oscillation period of the spicule is about $130 \mathrm{~s}$ with the amplitude of 1000 $\mathrm{km}$. A clear oscillation feature can be seen during $260 \mathrm{~s}$ which is nearly half of its total duration. It is noted that after the first $260 \mathrm{~s}$, the oscillation of the spicule was stopped. As we mentioned in the previous section, from Figure 2 we cannot determine the exact wavelength of spicule oscillation. In order to address the wavelength of spicule oscillation, we examined the phase of the oscillation over long distance (about $7500 \mathrm{~km}$ ) at different positions along the spicule axis. Thus we found that all oscillations are in phase (see Figure 3 ). So, the wavelength will be longer than $7500 \mathrm{~km}$. In addition, since the time cadence of SOT BFI data that we used in this study is $16 \mathrm{~s}$, the uncertainty in phase of the oscillation is about $1 / 8$ of the oscillation period of this spicule $(130 \mathrm{~s})$. Finally, the minimum wavelength is estimated to be $8 \times 7500 \mathrm{~km} \sim 60000 \mathrm{~km}$, at least (see Okamoto et al. 2007). The wave speed can be estimated to be about $460 \mathrm{~km} \mathrm{~s}^{-1}$. As a spicule density, we adopt the result of Beckers (1968) who determined that the spicule densities ranges from $2.2 \times 10^{-11}$ to $4.0 \times 10^{-10}$ $\mathrm{kg} \mathrm{m}^{-3}$. Then finally we can estimate the magnetic field strength to be 18-76 G. 


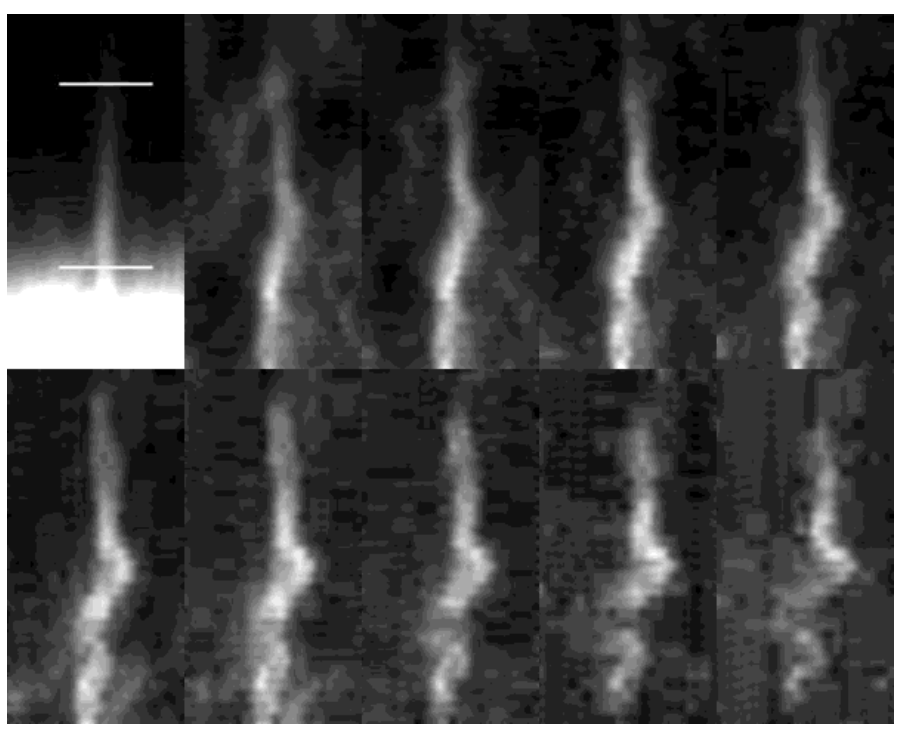

Fig. 3.- Time-slice images at different positions over $7500 \mathrm{~km}$ (distance between two horizontal white lines in the upper left image) along the spicule axis. The axes in $\mathrm{x}$ - and $\mathrm{y}$-direction for each time-slice image are the same as those of Figure 2.

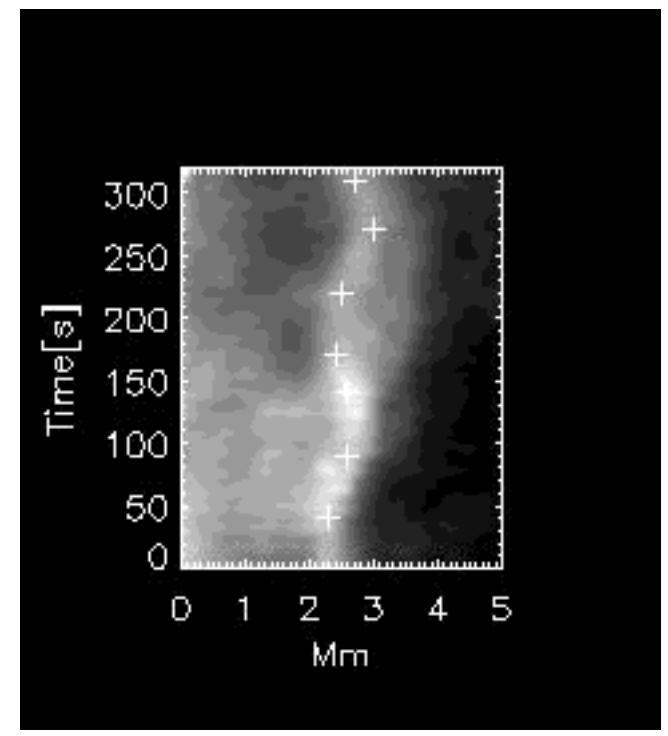

Fig. 4. - Time-slice image at the fixed position $(5000 \mathrm{~km}$ height) on the spicule axis. The spicule occurred at 23:33 UT on 2008 June 04.

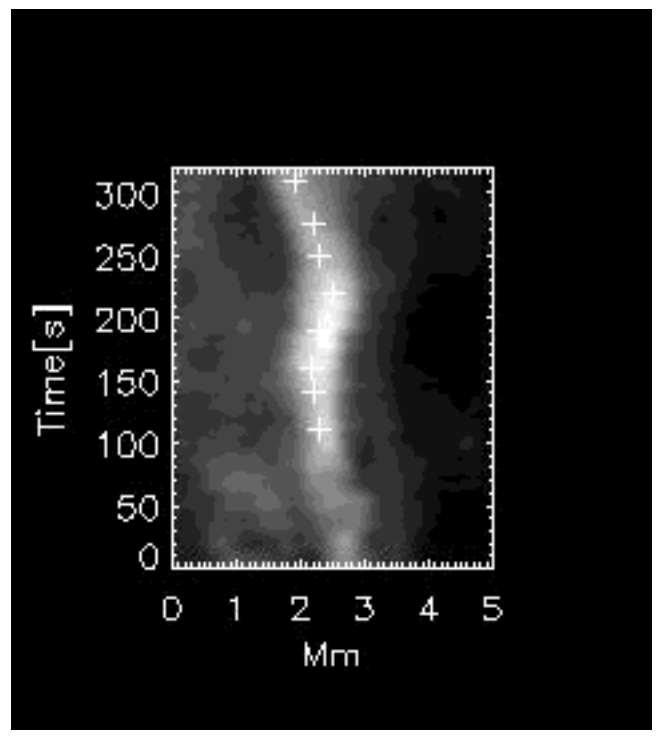

Fig. 5. - Time-slice image at the fixed position $(4200 \mathrm{~km}$ height) on the spicule axis. The spicule occurred at 23:42 UT on 2008 June 04. 
In addition, in Figure 2 we can see the intensity variations of spicule oscillation feature with time. It was first steady until around $300 \mathrm{~s}$ and then abruptly decreased. It is noted that there were only oscillations while the intensity is high. Thus we can think of a triggering process with heating source (like reconnection).

\section{(b) Spicule 2 (2008 June 04 23:33 UT)}

The spicule was occurred during from 23:33:33 UT to $23: 38: 52$ UT on 2008 June 04 in the solar north pole. Figure 4 shows the time-slice image of the spicule at the height of $5000 \mathrm{~km}$. The total duration of the spicule is about $320 \mathrm{~s}$ and the period of spicule oscillation is $180 \mathrm{~s}$ with the amplitude of $700 \mathrm{~km}$. In this case, the estimated minimum wavelength is about $56000 \mathrm{~km}$ and the wave speed is estimated to be about $310 \mathrm{~km} \mathrm{~s}^{-1}$. We also estimate the magnetic field strength to be 12 $52 \mathrm{G}$ for this spicule. It is noted that this event has the similar oscillation period with the well-known 3-min oscillation in the chromosphere.

\section{(c) Spicule 3 (2008 June 04 23:42 UT)}

This spicule was observed during from 23:42:04 UT to 23:47:09 UT on 2008 June 04 in the north polar region of the Sun. Figure 5 shows the time-slice image of the spicule at the height of $4200 \mathrm{~km}$. The spicule duration is about $305 \mathrm{~s}$ and the period of spicule oscillation is $170 \mathrm{~s}$ with the amplitude of $800 \mathrm{~km}$. The minimum wavelength and the wave speed of the spicule were estimated to be about $45000 \mathrm{~km}$ and about $260 \mathrm{~km} \mathrm{~s}^{-1}$ respectively. In this case, the estimated magnetic filed strength is $10-43 \mathrm{G}$.

\section{SUMMARY AND DISCUSSION}

In this study, we estimated the magnetic field strengths of three oscillating spicules observed in $\mathrm{Ca}$ II $\mathrm{H}$ line data of the Hinode SOT, based on the MHD coronal seismology technique. This is the first attempt to estimate the spicule magnetic field using observed MHD waves by the Hinode SOT.

(1) We observed three oscillating spicules in 2008 June. The observed oscillation properties are as follows: the total durations of spicules are 300-430 s, the oscillation period is 2-3 minutes, the transverse displacement from the spicule axis is $700-1000 \mathrm{~km}$, the transverse velocity is $8-15 \mathrm{~km} \mathrm{~s}^{-1}$, the estimated minimum wavelength is $45000-60000 \mathrm{~km}$, and the estimated wave speed is $260-460 \mathrm{~km} \mathrm{~s}^{-1}$. Being compared with the ground-based spectroscopic observation by Kukhianidze et al. (2006) and Zaqarashvili et al. (2007), our results were more than one order longer in wavelength, a factor of 3 or 4 larger in wave speed, and 2-3 times longer in period. This imply that the source of oscillation be quite different from that they suggested. They suggested that the source of observed kink waves is the dynamic motion of granular cell.
(2) We estimated the spicule magnetic field for nonstratified cases. In this estimation, we adopt the spicule density as $2.2 \times 10^{-11}-4.0 \times 10^{-10} \mathrm{~kg} \mathrm{~m}^{-3}$ from the Beckers (1968). The estimated magnetic filed strength is $10-18 \mathrm{G}$ for the lower density limit and $43-76 \mathrm{G}$ for upper density limit, respectively. In the previous studies, Zaqarashvili et al. (2007) and Singh \& Dwivedi (2007) estimated the magnetic field strength in spicules using the observed kink waves from ground-based observation as $12-15 \mathrm{G}$ and $12-22 \mathrm{G}$, respectively. Our result for the lower density limit is quite consistent with the previous estimations. But that for upper density limit is much larger than the usual magnetic field strength in spicules obtained from the previous studies. According to a recent research by Singh \& Dwivedi (2007), the spicule density is similar to the upper limit of Beckers (1968). Probably the difference in magnetic field strength seems come from the estimated wave speed. Also the wave speed strongly depends on the estimated wavelength of observed MHD waves. Thus regarding this issue, the reliable wavelength information is the most important factor to estimate the magnetic field strength in spicules.

(3) In this analysis, we estimated spicule magnetic field assuming that the observed spicule oscillations are propagating MHD kink waves. However, we could not determine the exact wavelengths of the spicule oscillations. We can only estimate the minimum wavelength of the oscillations, such as $60000 \mathrm{~km}, 56000 \mathrm{~km}$, and $45000 \mathrm{~km}$. This may be due to the short length of spicules as well as the longer wavelength of spicule oscillations. In fact this is the most weak point of this kind of analysis. There is another possibility to interpretation of this result. In this study, we found no spicule event showing any phase difference over heights. The real nature of spicule oscillation might be standing waves, not propagating waves. It may be a very important property of spicule oscillations and more investigations should be carried out. Moreover, Erdélyi et al. (2007) showed that, through a numerical simulation for a two-dimensional non-magnetic solar atmosphere, the photospheric 5-min driven waves can generate standing waves in the chromospheric cavity between the photospheric temperature minimum and the chromospheretransition region boundary. Regarding this issue, we will carefully examine spicule oscillations for a large sample of data near future.

(4) We should note that in the first event the spicule oscillation exists during the limited time (about $250 \mathrm{~s}$ ), although the lifetime of the spicule is much longer. Is there some strong damping mechanism ? This question seems to be beyond the scope of this study. At present stage, we can just speculate that if this is the damping case, the triggering mechanism of spicule oscillation may be impulsive (like low atmospheric reconnection), not continuous. We will also search for the cause of this strong damping on the spicule oscillation in future works.

In addition, according to Singh \& Dwivedi (2007) 
the effect of gravitational stratification give an error around $30 \%$ to estimate the magnetic field strength. Thus it is necessary to consider the gravitational stratification in spicule for this kind of analysis. The effect of gravitational stratification will be considered in our future work.

\section{ACKNOWLEDGEMENTS}

We are very thankful to the referee and Dr. Jongchul Chae for their helpful comments. This work has been supported by the "Development of Korean Space Weather Center" of KASI and the KASI basic research fund. Hinode is a Japanese mission developed and launched by ISAS/JAXA, collaborating with NAOJ as a domestic partner, NASA and STFC (UK) as international partners. Scientific operation of the Hinode mission is conducted by the Hinode science team organized at ISAS/JAXA. This team mainly consists of scientists from institutes in the partner countries. Support for the post-launch operation is provided by JAXA and NAOJ (Japan), STFC (U.K.), NASA (U.S.A.), ESA, and NSC (Norway). This work was (partly) carried out at the NAOJ Hinode Science Center, which is supported by the Grant-in-Aid for Creative Scientific Research "The Basic Study of Space Weather Prediction" from MEXT, Japan (Head Investigator: K. Shibata), generous donations from Sun Microsystems, and NAOJ internal funding.

\section{REFERENCES}

Aschwanden, M. J., Fletcher, L., Schrijver, C. J., \& Alexander, D., 1999, Coronal Loop Oscillations Observed with the Transition Region and Coronal Explorer, ApJ, 520, 880

Beckers, J. M., 1968, Solar Spicules (Invited Review Paper), Sol. Phys., 3, 367

Cirtain, J. W., et al., 2007, Evidence for Alfvén Waves in Solar X-ray Jets, Science, 318, 1580

De Moortel, I., Ireland, J., \& Walsh, R. W., 2000, Observation of oscillations in coronal loops , A\&A, 335, L23

De Pontieu, B., et al., 2007, Chromospheric Alfvénic Waves Strong Enough to Power the Solar Wind, Science, 318, 1574

De Pontieu, B., Erdelyi, R., \& de Wijn, A. G., 2003, Intensity Oscillations in the Upper Transition Region above Active Region Plage, ApJ, 595, L63

Deforest, C. E. \& Gurman, J. B., 1998, Observation of Quasi-periodic Compressive Waves in Solar Polar Plumes, ApJ, 501, L217

Edwin, P. M., \& Roberts, B., 1983, Wave propagation in a magnetic cylinder, Sol. Phys., 88, 179

Erdélyi, R., Malins, C., Tóth, G., \& de Pontieu, B., 2007, Leakage of photospheric acoustic waves into non-magnetic solar atmosphere, A\&A, 467, 1299
Hasan, S. S. \& Kalkofen, W., 1999, Excitation of Oscillations in Photospheric Flux Tubes through Buffeting by External Granules, ApJ, 519, 899

Hollweg, J. V., 1981, Alfvén waves in the solar atmosphere. II - Open and closed magnetic flux tubes, Sol. Phys., 70, 25

Kosugi, T. et al., 2007, The Hinode (Solar-B) Mission: An Overview, Sol. Phys., 243, 3

Kukhianidze, V., Zaqarashvili, T. V.; \& Khutsishvili, E. 2006, Observation of kink waves in solar spicules, A\&A, 449, L35

Kulidzanishvili, V. I. \& Zhugzhda, Iu. D., 1983, On the problem of spicular oscillations, Sol. Phys., 88, 35

Nakariakov, V. M. \& Ofman, L., 2001, Determination of the coronal magnetic field by coronal loop oscillations, A\&A, 372, L53

Nakariakov, V. M., Ofman, L., Deluca, E. E., Roberts, B., \& Davila, J. M., 1999, TRACE observation of damped coronal loop oscillations: Implications for coronal heating, Science, 285, 862

Nikolsky, G. M. \& Platova, A. G., 1971, Motions of $\mathrm{H}_{\alpha}$-spicules along the solar limb, Sol. Phys., 18, 403

Ofman, L., Nakariakov, V. M., \& Deforest, C. E., 1999, Slow Magnetosonic Waves in Coronal Plumes, ApJ, 514,441

Okamoto, T. J., et al., 2007, Coronal Transverse Magnetohydrodynamic Waves in a Solar Prominence, Science, 318, 1577

Robbrecht, E., Verwichte, E., Berghmans, D., Hochedez, J. F., Poedts, S., \& Nakariakov, V. M., 2001, Slow magnetoacoustic waves in coronal loops: EIT and TRACE, A\&A, 370, 591

Roberts, B., 1979, Spicules - The resonant response to granular buffeting, Sol. Phys., 61, 23

Roberts, B., 2004, MHD Waves in the Solar Atmosphere, In Proceedings of 'SOHO 13 - Waves, Oscillations and Small-Scale Transient Events in the Solar Atmosphere: A Joint View from SOHO and TRACE', Palma de Mallorca, Balearic Islands, Spain , ESA SP-547, 1

Shimizu, T. et al,. 2008, Image Stabilization System for Hinode (Solar-B) Solar Optical Telescope, Sol. Phys., 249, 221

Singh, K. A. P. \& Dwivedi, B. N., 2007, Estimation of spicule magnetic field using observed kink waves, New Astronomy, 12, 479

Spruit, H. C., 1981, Motion of magnetic flux tubes in the solar convection zone and chromosphere, A\&A, 98,155

Sterling, Alphonse C., 2000, Solar Spicules: A Review of Recent Models and Targets for Future Observations - (Invited Review), Sol. Phys., 196, 79 
Suematsu, Y., 1998, Solar Spicules: A brief review of recent high-resolution observations, In Proceedings of an International meeting: Solar Jets and Coronal Plumes, Guadeloupe, France, ESA SP-421, p.19

Suematsu, Y., et al., 2008, The Solar Optical Telescope of Solar-B (Hinode): The Optical Telescope Assembly, Sol. Phys., 249, 197

Tsuneta, S., et al., 2008, The Solar Optical Telescope for the Hinode Mission: An Overview, Sol. Phys., 249, 167

Xia, L. D., Popescu, M. D., Doyle, J. G., \& Giannikakis, J., 2005, Time series study of EUV spicules observed by SUMER/SoHO, A\&A, 438, 1115

Zaqarashvili, T. V., Khutsishvili, E., Kukhianidze, V., \& Ramishvili, G., 2007, Doppler-shift oscillations in solar spicules, A\&A, 474, 627 